\title{
Patterns of alcohol policy enforcement activities among local law enforcement agencies: A latent class analysis
}

\author{
Darin J. Erickson ${ }^{1}$, Patricia C. Rutledge ${ }^{2}$, Kathleen M. Lenk ${ }^{1}$, Toben F. Nelson ${ }^{1}$, Rhonda Jones-Webb ${ }^{1}$, and \\ Traci L. Toomey ${ }^{1}$ \\ ${ }^{1}$ Division of Epidemiology and Community Health, School of Public Health, University of Minnesota, Minneapolis, MN \\ ${ }^{2}$ Department of Psychology, Allegheny College, Meadville, PA
}

\begin{abstract}
Aims: We assessed levels and patterns of alcohol policy enforcement activities among U.S. local law enforcement agencies.

Design/Setting/Participants: We conducted a cross-sectional survey of a representative sample of 1,631 local law enforcement agencies across the 50 states.

Measures/Methods: We assessed 29 alcohol policy enforcement activities within each of five enforcement domains-underage alcohol possession/consumption, underage alcohol provision, underage alcohol sales, impaired driving, and overservice of alcohol—and conducted a series of latent class analyses to identify unique classes or patterns of enforcement activity for each domain.

Findings: We identified three to four unique enforcement activity classes for each of the enforcement domains. In four of the domains, we identified a Uniformly Low class (i.e., little or no enforcement) and a Uniformly High enforcement activity class (i.e., relatively high levels of enforcement), with one or two middle classes where some but not all activities were conducted. The underage provision domain had a Uniformly Low class but not a Uniformly High class. The Uniformly Low class was the most prevalent class in three domains: underage provision (58\%), underage sales (61\%), and overservice (79\%). In contrast, less than a quarter of agencies were in Uniformly High classes.

Conclusions: We identified qualitatively distinct patterns of enforcement activity, with a large proportion of agencies in classes characterized by little or no enforcement and fewer agencies in high enforcement classes. An important next step is to determine if these patterns are associated with rates of alcohol use and alcohol-related injury and mortality.
\end{abstract}

Numerous policies that regulate the use and sale of alcohol, such as the minimum legal drinking age and limits on blood alcohol content (BAC) among drivers, reduce negative consequences associated with alcohol use (Voas, Tippetts, \& Fell, 2003; Wagenaar \& Toomey, 2002); however, policies need to be actively enforced to be maximally effective (National Research Council \& Institute of Medicine, 2004; Ross, 1984). Deterrence theory suggests that individuals are more likely to comply with laws if they perceive a high certainty of being apprehended and penalized when laws are violated, and if penalties are swiftly applied and severe (Gibbs, 1975; Ross, 1984; Tittle, 1980). Although several studies have examined alcohol policy enforcement activities, these studies have generally examined a single type of enforcement, such as impaired driving enforcement, rather than the range of possible enforcement activities.
A number of individual alcohol policy enforcement activities have been shown to be effective. Compliance checks, where an underage person supervised by law enforcement attempts to purchase alcohol, have been shown to be effective in reducing illegal sales of alcohol to underage patrons (Grube, 1997; Wagenaar, Toomey, \& Erickson, 2005). Sobriety checkpoints, where a roadblock is set up to evaluate motor vehicle drivers for alcohol use, have been shown to be effective at reducing drinkingdriving incidents by increasing both the actual and perceived certainty of being penalized (Elder et al., 2002; Ferguson, 2012).

Although the effectiveness of some individual enforcement activities has been established, research has not examined how different types of alcohol enforcement activities may be combined to be optimally effective. Possible reasons for this are the lack of readily available enforcement data, as

Correspondence: Darin J. Erickson, PhD, 1300 S. Second St. Suite 300, Minneapolis, MN 55454. Telephone: 612-626-0516; Fax: 612-624-0315; Email: erick232@umn.edu

Financial support: This study was supported by a grant from the National Institute of Alcohol Abuse and Alcoholism (R01 AA017873; Darin J. Erickson, Principal Investigator)

Keywords: Alcohol, Enforcement, Latent Class Analysis 
well as the lack of developed methods for appropriately combining different types of alcohol enforcement (e.g., underage alcohol use, impaired driving). To address these limitations, we collected alcohol-related enforcement data through a U.S. survey of a representative sample of local law enforcement agencies within each state. We assessed levels and patterns of alcohol policy enforcement among these agencies within each of five enforcement domainsunderage alcohol possession/consumption, underage alcohol provision, underage alcohol sales, impaired driving, and overservice of alcohol. We also examined agency- and community-level variables associated with enforcement patterns within each domain.

\section{Methods}

We conducted a survey of a stratified random sample of local law enforcement agencies in 2010-2011 regarding their alcohol enforcement efforts. This study received an Institutional Review Board (IRB) exemption by the University of Minnesota IRB.

\section{Sample}

We used a multi-stage sampling strategy to select agencies using a list of 15,833 municipal and county agencies from the U.S. Bureau of Justice Statistics for 2004 (the most recent list available at time of survey). First, we divided the 50 states into two groups of 25 (small vs. large) using the median number of agencies per state (300). Hawaii had only four agencies so we included all four in our sample. For the other 49 states, we sampled based on the proportion of county sheriff versus municipal police per state, ensuring equal numbers of small and large agencies (using median number of officers per agency). We selected 20 agencies in small states and 40 agencies in large states for a total of $1,484(4+[24 \times 20]+[25 \times 40])$. Additionally, Texas has a unique type of agency called a "constable"-we randomly selected 20 of these 512 agencies, which brought our sample to 1,504 agencies. Finally, given that this sampling strategy did not necessarily include agencies in the largest cities (which tend to account for a high percentage of a state's population), we added the municipal police agency from the three largest cities in each state if these agencies were not already in our sample $(n=127)$. Our final sample was 1,631 law enforcement agencies.

\section{Survey Administration}

At each agency, we attempted to survey the officer most knowledgeable about the agency's enforcement activities pertaining to alcohol-related laws. We initially conducted the survey via telephone. The survey was administered by trained research staff who entered responses into an online data collection form. If requested by the respondent, we provided the option of completing the survey online (the survey link was sent via email; $47 \%$ of participants completed the survey online). In addition, 10 agencies completed the survey by regular mail or fax; these data were entered into an online form by research staff. Data were housed on a secure server and were maintained according to standards for Internet security and research protection established by the University of Minnesota IRB.

\section{Response Rate}

The response rate was $66.3 \%$ (1082 out of 1631). Among the three largest agencies per state $(n=150), 39 \%$ (58) did not respond. In two states (Indiana and New Jersey) none of the three responded. Agencies that did not respond to our survey were not significantly different $(p>.05)$ from agencies that did respond in terms of agency type, number of agencies in the state, number of officers per 1000 residents, proportion of residents living in poverty, or the proportion of Black residents in the jurisdiction. However, agencies in smaller jurisdictions (population $<10,000$ ) and agencies in jurisdictions with a lower proportion of Hispanic residents $(<3 \%$ ) were less likely to respond.

\section{Measures}

\section{Enforcement activities}

We measured 29 enforcement activities within five broad domains of alcohol enforcement (Table 1): (1) underage possession/consumption (e.g., party patrols); (2) underage provision (e.g., citations for hosts of underage parties); (3) underage sales (compliance checks); (4) impaired driving (e.g., sobriety checkpoints); and (5) overservice of alcohol at alcohol establishments (e.g., random inspections). We grouped enforcement activities into domains to facilitate analyses and interpretation. The underage possession/consumption, provision, and sales domains each had six dichotomous indicators; the impaired driving domain had four; and the overservice domain had seven.

\section{Agency characteristics}

We measured two agency characteristics from the survey: number of officers (continuous measure) and whether any full-time officers were assigned primarily to enforcement of alcohol-related laws (yes/no). For number of officers, we created a ratio-number of officers per 1000 residents in the agency's jurisdiction.

\section{Community characteristics}

We obtained measures of characteristics of the communities for which each agency had jurisdiction from the 2010 U.S. Census and the enforcement survey. Censusbased variables included total population (used for number of officers per population), percent living in poverty, percent Black, and percent Hispanic. We also included a measure designating regions of the country based on alcohol consumption levels: dry, moderate, or wet as defined by Kerr (2010). Finally, we included measures of law enforcement perceptions of how common three problems are in their community: underage drinking, impaired driving, and overservice of alcohol $(1=$ not common, 2 = somewhat common, 3 = very common).

\section{Analytic Strategy}

We first assessed descriptive statistics for each measure. We then conducted a series of latent class analyses (LCAs) to identify unique classes or patterns of enforcement activity separately for indicators in each of the five 
domains. For each domain, models were estimated with number of classes ranging from 2 to 5 (or until the model failed to converge). We used a number of standard criteria (Collins \& Lanza, 2010) to facilitate model choice, including goodness of fit (Akaike Information Criteria [AIC] and Bayesian Information Criteria [BIC]), homogeneity (the extent to which agencies within classes look similar), separation (the extent to which classes are distinct), the sample sizes of the individual classes (not wanting to extract classes with only one or a small number of agencies), and interpretability. Item-response probabilities were used for class interpretation, with high or low probabilities (more than $\sim 70 \%$ or less than $\sim 30 \%$, respectively) indicating reasonable homogeneity. Once an optimal number of classes was determined, each agency was assigned to its most likely class.

Following estimation of an optimal number of classes, conditional analyses were conducted to examine correlates of class. For each of the five domains, class was regressed separately on all agency and community characteristics using multinomial logistic regression (the measures indicating whether underage drinking, impaired driving, and overservice were common were included in models for the corresponding domains). All significant independent variables were then modeled simultaneously in a multivariate multinomial logistic regression separately for each of the five domains.

All LCAs were conducted in Mplus Version 7 (Muthén \& Muthén, 1998-2012). Maximum likelihood was used to accommodate the small amount of missing data for indicators. Multinomial logistic regressions were conducted using PROC LOGISTIC in SAS Version 9.3 (SAS Institute, Inc., 2011).

\section{Results}

Descriptive statistics for the 29 enforcement activities by domain are presented in Table 1 . The most common activities in the underage possession/consumption domain were party patrols and field patrols $(58.7 \%$ and $57.1 \%$, respectively). The most common activities in the underage provision domain were arrests or citations for hosts of underage parties (30.5\%) and educational efforts (25.1\%). In the underage sales domain, $39.4 \%$ of agencies reported conducting compliance checks. The most common activities in the impaired driving domain were saturation patrols (65.9\%) and media messages (65.9\%). Lastly, the most common activities in the overservice domain were walk-throughs (21.4\%) and random inspections (18.3\%). Table 1 also presents descriptive statistics for agency and community characteristics.

\section{Latent Class Analyses}

The results of the LCAs in each of the five enforcement domains are presented in Table 2, which provides the itemresponse probabilities for the selected solutions (high likelihood of enforcement indicated by item-response probabilities more than $\sim 70 \%$ and low likelihood of enforcement indicated by probabilities less than $\sim 30 \%$ ). In the underage possession/consumption domain, the fourclass solution was selected because it had the lowest BIC and AIC, as well as good item separation, adequate class size for all classes, and a high degree of interpretability. In the underage provision domain, the three-class solution was selected because it had the lowest BIC and AIC, as well as good item separation, adequate class size for all classes, and a high degree of interpretability. In the underage sales domain, the four-class solution was selected because, although it had the second lowest BIC, it had the lowest AIC and it was characterized by good item separation, adequate class sizes, and it was highly interpretable. Similarly, in the overservice domain, the four-class solution was selected because, although it had the second lowest BIC, it had the lowest AIC and it was characterized by good item separation, adequate class sizes, and it was highly interpretable. Lastly, in the impaired driving domain, the three-class solution was selected because it had good item separation, adequate class sizes, and a high degree of interpretability. In this domain, the fit indices for this three-class solution were not as low as in the two-class solution; however, the fit for both solutions was similar and the three-class solution had a greater degree of interpretability.

Descriptions of each class and proportion of agencies per class are also included in Table 2 for each of the five domains. Four of the domains had a Uniformly Low class (few or no enforcement activities) and a Uniformly High class (all or most activities), and one or two middle classes where some but not all activities were conducted. The underage provision domain had a Uniformly Low class but not a Uniformly High class. The Uniformly Low class was the most prevalent class in the underage provision (57.9\%), underage sales (60.6\%), and overservice $(78.7 \%)$ domains. The most prevalent class in the underage possession/consumption domain (47.4\%) was one of the middle classes, characterized by a high likelihood of conducting party patrols and field patrols. In the impaired driving domain, the most prevalent class (50.9\%) was the middle class, characterized by a high likelihood of conducting saturation patrols and using media messages.

\section{Multinomial Logistic Regression}

The results of the multinomial logistic regression analyses for each of the five domains are presented in Table 3. The Uniformly Low class was used as the reference group for each domain. The consistent findings in this set of analyses were that (1) having an officer specifically assigned to alcohol enforcement was associated with being in a higher (vs. lower) enforcement class across all five domains; and (2) community characteristics from the U.S. Census were mostly nonsignificant. Perceiving underage drinking, impaired driving, or overservice as being common was associated with being in a higher class (except for in the underage sales domain). Being located in a wet versus dry region was associated with being in a higher class for the underage possession/consumption, underage sales, and impaired driving domains. 
Table 1

Descriptive statistics

\begin{tabular}{|c|c|c|}
\hline Enforcement Activities & $N$ & $\%$ or $M(S D)$ \\
\hline \multicolumn{3}{|l|}{ Underage Possession/Consumption } \\
\hline Party Patrols & 956 & $58.7 \%$ \\
\hline Field Patrols & 935 & $57.1 \%$ \\
\hline Random Inspections & 908 & $42.8 \%$ \\
\hline Fake ID Use Arrests/Citations & 1065 & $27.9 \%$ \\
\hline Cops in Shops & 874 & $11.3 \%$ \\
\hline Other Possession/Consumption Efforts & 845 & $24.3 \%$ \\
\hline \multicolumn{3}{|l|}{ Underage Provision } \\
\hline Hosts of Underage Parties: Arrests/Citations & 1005 & $30.5 \%$ \\
\hline Education Efforts & 1056 & $25.1 \%$ \\
\hline Sticker Campaigns (on Alcohol Products) & 1056 & $9.8 \%$ \\
\hline Shoulder Tap & 1056 & $9.3 \%$ \\
\hline Keg Registration Arrests/Citations & 965 & $5.4 \%$ \\
\hline Other Provision Efforts & 1056 & $15.9 \%$ \\
\hline \multicolumn{3}{|l|}{ Underage Sales } \\
\hline Conduct Compliance Checks & 1070 & $39.4 \%$ \\
\hline At All On-Premise Outlets & 1064 & $25.9 \%$ \\
\hline At All Off-Premise Outlets & 1064 & $24.5 \%$ \\
\hline Twice a Year or More & 1042 & $19.3 \%$ \\
\hline Conduct Follow-Up Checks & 1065 & $30.0 \%$ \\
\hline Follow Up within 3 Months & 964 & $11.8 \%$ \\
\hline \multicolumn{3}{|l|}{ Impaired Driving } \\
\hline Saturation Patrols & 942 & $65.9 \%$ \\
\hline Media Messages & 1004 & $65.9 \%$ \\
\hline Sobriety Checkpoints & 906 & $44.0 \%$ \\
\hline Open Container Enforcement & 1038 & $43.0 \%$ \\
\hline \multicolumn{3}{|l|}{ Overservice (at Alcohol Establishments) } \\
\hline Walk-throughs & 1021 & $21.4 \%$ \\
\hline Random Inspections & 1021 & $18.3 \%$ \\
\hline Media Messages & 1005 & $16.5 \%$ \\
\hline Observations & 1011 & $15.9 \%$ \\
\hline Last Call Enforcement & 995 & $9.4 \%$ \\
\hline BAC Testing & 1001 & $6.8 \%$ \\
\hline Pseudo Intoxicated Purchase Attempts & 998 & $2.1 \%$ \\
\hline \multicolumn{3}{|l|}{ Agency and Community Characteristics } \\
\hline Total Population & 1077 & $72,021(33,1785)$ \\
\hline Percent Black & 1077 & $8.7 \%(15.4)$ \\
\hline Percent Hispanic & 1077 & $9.3 \%(14.3)$ \\
\hline Percent Poverty & 1076 & $15.0 \%(8.9)$ \\
\hline Officers per 1000 Population & 1075 & $3.3(21.8)$ \\
\hline Officer Assigned to Alcohol Enforcement & 1062 & $27.9 \%$ \\
\hline Underage Drinking Common & 1043 & $2.33(0.58)$ \\
\hline Overservice of Alcohol Common & 1011 & $1.67(0.65)$ \\
\hline Alcohol-Impaired Driving Common & 1038 & $2.23(0.55)$ \\
\hline Region & 1077 & \\
\hline Wet (North Central/New England) & & $40.0 \%$ \\
\hline Moderate (Mid-Atlantic, Pacific, South Coast) & & $33.7 \%$ \\
\hline Dry (South) & & $26.3 \%$ \\
\hline
\end{tabular}


Table 2

Latent class analyses by domain: Item-response probabilities

\begin{tabular}{|c|c|c|c|c|}
\hline Underage Possession/Consumption & $\begin{array}{c}\text { Uniformly Low } \\
(26.3 \%)\end{array}$ & $\begin{array}{c}\text { Fake ID } \\
(4.1 \%)\end{array}$ & $\begin{array}{c}\text { Party \& Field Patrols } \\
(47.4 \%)\end{array}$ & $\begin{array}{c}\text { Uniformly High } \\
(22.2 \%)\end{array}$ \\
\hline Party Patrols & 0.00 & 0.37 & 0.85 & 0.91 \\
\hline Field Patrols & 0.03 & 0.08 & 0.86 & 0.89 \\
\hline Random Inspections & 0.07 & 0.36 & 0.43 & 1.00 \\
\hline Cops in Shops & 0.00 & 0.27 & 0.03 & 0.48 \\
\hline Fake ID Use Arrests/Citations & 0.07 & 0.68 & 0.22 & 0.53 \\
\hline Other Possession/Consumption & 0.08 & 0.60 & 0.11 & 0.71 \\
\hline Underage Provision & $\begin{array}{c}\text { Uniformly Low } \\
(57.9 \%)\end{array}$ & $\begin{array}{l}\text { Hosts of Underage } \\
\text { Parties (28.4\%) } \\
\end{array}$ & $\begin{array}{c}\text { Education } \\
(13.7 \%)\end{array}$ & \\
\hline $\begin{array}{l}\text { Hosts of Underage Parties } \\
\text { Arrests/Citations }\end{array}$ & 0.00 & 1.00 & 0.48 & \\
\hline Education Efforts & 0.07 & 0.17 & 1.00 & \\
\hline $\begin{array}{l}\text { Sticker Campaigns (on Alcohol } \\
\text { Products) }\end{array}$ & 0.01 & 0.05 & 0.48 & \\
\hline Shoulder Tap Enforcement & 0.04 & 0.12 & 0.26 & \\
\hline Keg Registration Arrests/Citations & 0.02 & 0.10 & 0.11 & \\
\hline Other Provision & 0.11 & 0.22 & 0.27 & \\
\hline Underage Sales & $\begin{array}{c}\text { Uniformly Low } \\
(\mathbf{6 0 . 6 \% )} \\
\end{array}$ & $\begin{array}{c}\text { Limited Checks } \\
(7.5 \%)\end{array}$ & $\begin{array}{c}\text { Checks with Follow- } \\
\text { up (6.4\%) } \\
\end{array}$ & $\begin{array}{c}\text { Uniformly High } \\
(25.6 \%)\end{array}$ \\
\hline Conduct Compliance Checks & 0.00 & 1.00 & 1.00 & 1.00 \\
\hline At All On-Premise Outlets & 0.00 & 0.05 & 0.21 & 1.00 \\
\hline At All Off-Premise Outlets & 0.00 & 0.19 & 0.16 & 0.92 \\
\hline Twice a Year or More & 0.00 & 0.22 & 0.69 & 0.54 \\
\hline Conduct Follow-Up Checks & 0.00 & 0.60 & 0.95 & 0.76 \\
\hline Follow Up within 3 Months & 0.00 & 0.00 & 0.62 & 0.35 \\
\hline Impaired Driving & $\begin{array}{c}\text { Uniformly Low } \\
(29.7 \%)\end{array}$ & $\begin{array}{c}\text { Saturation Patrols \& } \\
\text { Media (50.9\%) } \\
\end{array}$ & $\begin{array}{c}\text { Uniformly High } \\
(19.4 \%)\end{array}$ & \\
\hline Saturation Patrols & 0.00 & 0.84 & 1.00 & \\
\hline Media Messages & 0.30 & 0.71 & 0.95 & \\
\hline Sobriety Checkpoints & 0.00 & 0.51 & 0.80 & \\
\hline Open Container Enforcement & 0.23 & 0.42 & 0.67 & \\
\hline Overservice (at Alcohol Establishments) & $\begin{array}{c}\text { Uniformly Low } \\
(\mathbf{7 8 . 7 \% )}\end{array}$ & $\begin{array}{c}\text { Walk-throughs \& } \\
\text { inspections (10.6\%) }\end{array}$ & $\begin{array}{c}\text { Multiple Strategies } \\
(5.0 \%) \\
\end{array}$ & $\begin{array}{c}\text { Uniformly High } \\
(5.7 \%)\end{array}$ \\
\hline Walk-throughs & 0.01 & 0.95 & 1.00 & 1.00 \\
\hline Random Inspections & 0.00 & 0.87 & 0.73 & 0.96 \\
\hline Observations & 0.00 & 0.57 & 1.00 & 0.98 \\
\hline Last Call Enforcement & 0.00 & 0.27 & 0.84 & 0.64 \\
\hline BAC Testing & 0.00 & 0.20 & 0.00 & 1.00 \\
\hline Pseudo Intoxicated Purchase Attempts & 0.00 & 0.05 & 0.03 & 0.33 \\
\hline Media Messages & 0.11 & 0.19 & 0.53 & 0.56 \\
\hline
\end{tabular}

Note. To aid in interpretation, item-response probabilities greater than or equal to .65 are bolded. 
Table 3

Multivariate multinomial logistic regression by domain: Odds Ratios and 95\% Confidence Intervals

\begin{tabular}{|c|c|c|c|c|c|}
\hline $\begin{array}{l}\text { Underage Possession/ } \\
\text { Consumption }\end{array}$ & Wald $X^{2}$ & $p$ & $\begin{array}{c}\text { Fake ID } \\
\text { OR }(95 \% \mathrm{CI})\end{array}$ & $\begin{array}{l}\text { Party \& Field Patrol } \\
\text { OR }(95 \% \text { CI })\end{array}$ & $\begin{array}{c}\text { Uniformly High } \\
\text { OR (95\% CI) }\end{array}$ \\
\hline Total Population & 6.11 & .11 & $1.00(1.00,1.00)$ & $1.00(1.00,1.00)$ & $1.00(1.00,1.00)$ \\
\hline Percent Black & 0.66 & .88 & $0.71(0.04,11.59)$ & $0.84(0.29,2.47)$ & $0.55(0.13,2.33)$ \\
\hline Percent Hispanic & 8.14 & $.04 *$ & $2.63(0.30,23.43)$ & $0.42(0.11,1.55)$ & $2.48(0.57,10.89)$ \\
\hline Percent Poverty & 4.02 & .26 & $0.24(0.00,20.98$ & $1.97(0.29,13.20)$ & $0.24(0.02,2.73)$ \\
\hline Officers per 1000 Population & 1.82 & .61 & $1.01(0.93,1.10)$ & $1.01(0.97,1.05)$ & $1.03(0.98,1.07)$ \\
\hline $\begin{array}{l}\text { Officer Assigned to Alcohol } \\
\text { Enforcement }\end{array}$ & 39.94 & $<.0001^{*}$ & $2.51(1.22,5.13)^{*}$ & $1.39(0.95,2.02)$ & $3.34(2.21,5.03)^{*}$ \\
\hline Underage Drinking Common & 24.18 & $<.0001^{*}$ & $1.15(0.65,2.03)$ & $1.73(1.33,2.25)^{*}$ & $2.03(1.47,2.80)^{*}$ \\
\hline Region: Wet & 23.10 & $.0008 *$ & $5.01(1.36,18.48)^{*}$ & $1.53(1.03,2.27)^{*}$ & $1.64(1.02,2.65)^{*}$ \\
\hline Region: Moderate & & & $7.96(2.18,29.07)^{*}$ & $1.82(1.20,2.76)^{*}$ & $1.08(0.63,1.84)$ \\
\hline \multicolumn{6}{|l|}{ Region: Dry (Reference) } \\
\hline Underage Provision & Wald $X^{2}$ & $p$ & $\begin{array}{c}\text { Hosts of Underage } \\
\text { Parties } O R(95 \% \mathrm{CI})\end{array}$ & $\begin{array}{c}\text { Education } \\
\text { OR }(95 \% \mathrm{CI})\end{array}$ & \\
\hline Total Population & 1.72 & .42 & $1.00(1.00,1.00)$ & $1.00(1.00,1.00)$ & \\
\hline Percent Black & 1.65 & .44 & $0.53(0.14,2.00)$ & $0.49(0.12,1.95)$ & \\
\hline Percent Hispanic & 2.65 & .27 & $2.66(0.75,9.37)$ & $2.01(0.49,8.31)$ & \\
\hline Percent Poverty & 17.08 & $.0002 *$ & $0.01(0.00,0.09)^{*}$ & $0.97(0.10,9.40)$ & \\
\hline Officers per 1000 Population & 4.40 & .11 & $1.03(1.00,1.07)$ & $0.99(0.93,1.05)$ & \\
\hline $\begin{array}{l}\text { Officer Assigned to Alcohol } \\
\text { Enforcement }\end{array}$ & 15.99 & $.0003^{*}$ & $1.83(1.30,2.57)^{*}$ & $1.77(1.20,2.60)^{*}$ & \\
\hline Underage Drinking Common & 9.34 & $.009 *$ & $1.50(1.14,1.97)^{*}$ & $1.30(0.95,1.77)$ & \\
\hline Region: Wet & 5.38 & .25 & $1.11(0.72,1.71)$ & $1.17(0.74,1.86)$ & \\
\hline Region: Moderate & & & $1.26(0.80,1.98)$ & $0.74(0.44,1.26)$ & \\
\hline \multicolumn{6}{|l|}{ Region: Dry (Reference) } \\
\hline Underage Sales & Wald $X^{2}$ & $p$ & $\begin{array}{c}\text { Limited Checks } \\
\text { OR (95\% CI) }\end{array}$ & $\begin{array}{c}\text { Checks with Follow-up } \\
\text { OR (95\% CI) }\end{array}$ & $\begin{array}{c}\text { Uniformly High } \\
\text { OR (95\% CI) }\end{array}$ \\
\hline Total Population & 6.76 & .08 & $1.00(1.00,1.00)$ & $1.00(1.00,1.00)$ & $1.00(1.00,1.00)$ \\
\hline Percent Black & 0.17 & .98 & $0.98(0.16,5.92)$ & $1.28(0.18,9.12)$ & $1.23(0.38,3.96)$ \\
\hline Percent Hispanic & 6.28 & .10 & $0.62(0.08,4.68)$ & $1.03(0.08,12.63)$ & $3.88(1.21,12.43)^{*}$ \\
\hline Percent Poverty & 3.36 & .34 & $0.60(0.02,15.27)$ & $0.17(0.01,5.83)$ & $0.18(0.02,1.33)$ \\
\hline Officers per 1000 Population & 2.96 & .40 & $0.95(0.85,1.06)$ & $1.03(0.99,1.06)$ & $1.01(0.98,1.04)$ \\
\hline $\begin{array}{l}\text { Officer Assigned to Alcohol } \\
\text { Enforcement }\end{array}$ & 39.01 & $<.0001^{*}$ & $2.56(1.52,4.28)^{*}$ & $2.76(1.59,4.78)^{*}$ & $2.45(1.77,3.41)^{*}$ \\
\hline Underage Drinking Common & 4.34 & .23 & $1.38(0.89,2.12)$ & $0.93(0.59,1.47)$ & $1.23(0.95,1.60)$ \\
\hline Region: Wet & 19.33 & $.004^{*}$ & $1.12(0.56,2.24)$ & $1.39(0.70,2.76)$ & $1.96(1.29,2.97)^{*}$ \\
\hline Region: Moderate & & & $1.67(0.85,3.28)$ & $0.80(0.37,1.75)$ & $1.09(0.70,1.72)$ \\
\hline \multicolumn{6}{|l|}{ Region: Dry (Reference) } \\
\hline Impaired Driving & Wald $X^{2}$ & $p$ & $\begin{array}{l}\text { Saturation Patrols \& } \\
\text { Media OR (95\% CI) }\end{array}$ & $\begin{array}{c}\text { Uniformly High } \\
\text { OR (95\% CI) }\end{array}$ & \\
\hline Total Population & 0.42 & .81 & $1.00(1.00,1.00)$ & $1.00(1.00,1.00)$ & \\
\hline Percent Black & 1.39 & .50 & $1.96(0.64,5.98)$ & $1.59(0.39,6.40)$ & \\
\hline Percent Hispanic & 1.66 & .44 & $2.11(0.63,7.05)$ & $2.23(0.48,10.35)$ & \\
\hline Percent Poverty & 9.92 & $.007 *$ & $0.05(0.01,0.34)^{*}$ & $0.09(0.01,0.99)^{*}$ & \\
\hline Officers per 1000 Population & 4.36 & .11 & $1.05(0.99,1.12)$ & $1.07(1.00,1.14)$ & \\
\hline $\begin{array}{l}\text { Officer Assigned to Alcohol } \\
\text { Enforcement }\end{array}$ & 54.85 & $<.0001^{*}$ & $2.51(1.70,3.69)^{*}$ & $5.46(3.48,8.55)^{*}$ & \\
\hline Impaired Driving Common & 8.89 & $.012 *$ & $1.38(1.05,1.81)^{*}$ & $1.67(1.17,2.36)^{*}$ & \\
\hline Region: Wet & 27.92 & $<.0001^{*}$ & $0.61(0.41,0.91)^{*}$ & $0.25(0.15,0.42)^{*}$ & \\
\hline Region: Moderate & & & $0.69(0.45,1.06)$ & $0.43(0.25,0.73)^{*}$ & \\
\hline Region: Dry (Reference) & & & & & \\
\hline
\end{tabular}




\begin{tabular}{|c|c|c|c|c|c|}
\hline Overservice & Wald $X^{2}$ & $p$ & $\begin{array}{c}\text { Walk Through \& } \\
\text { Inspect } \\
\text { OR }(95 \% \mathrm{CI}) \\
\end{array}$ & $\begin{array}{c}\text { Multiple Strategies } \\
\text { OR (95\% CI) } \\
\end{array}$ & $\begin{array}{c}\text { Uniformly High } \\
\text { OR }(95 \% \text { CI) }\end{array}$ \\
\hline Total Population & 4.06 & .25 & $1.00(1.00,1.00)$ & $1.00(1.00,1.00)$ & $1.00(1.00,1.00)$ \\
\hline Percent Black & 3.63 & .30 & $0.17(0.02,1.31)$ & $0.64(0.07,6.18)$ & $0.31(0.03,3.55)$ \\
\hline Percent Hispanic & 7.98 & $.046 *$ & $5.92(1.39,25.46)^{*}$ & $5.65(0.80,40.01)$ & $3.49(0.37,32.51)$ \\
\hline Percent Poverty & 2.05 & .56 & $0.36(0.02,6.01)$ & $1.53(0.04,66.48)$ & $0.08(0.00,4.31)$ \\
\hline Officers per 1000 Population & 1.23 & .75 & $0.97(0.90,1.05)$ & $1.02(0.98,1.06)$ & $1.00(0.94,1.05)$ \\
\hline $\begin{array}{l}\text { Officer Assigned to Alcohol } \\
\text { Enforcement }\end{array}$ & 31.04 & $<.0001^{*}$ & $1.42(0.90,2.23)$ & $2.68(1.50,4.79)^{*}$ & $3.86(2.20,6.76)^{*}$ \\
\hline Overservice Common & 6.65 & .08 & $1.09(0.80,1.50)$ & $1.40(0.92,2.15)$ & $1.57(1.05,2.36)^{*}$ \\
\hline Region: Wet & 3.32 & .77 & $1.04(0.59,1.83)$ & $1.59(0.70,3.59)$ & $0.70(0.34,1.43)$ \\
\hline Region: Moderate & & & $0.94(0.51,1.74)$ & $1.09(0.44,2.67)$ & $0.63(0.28,1.41)$ \\
\hline Region: Dry (Reference) & & & & & \\
\hline
\end{tabular}

Note. ${ }^{*}=p<.05 ; O R=$ odds ratio; $C I=$ confidence interval; Reference category $=$ Uniformly Low.

\section{Discussion}

The current study has a number of important findings. First and foremost the study provides evidence that alcohol enforcement activities cluster in meaningful ways and that enforcement agencies can be classified within each domain based on the pattern or types of alcohol enforcement activities they conduct. A latent class approach provides one way for moving beyond simply counting then summing the number of enforcement activities. With the simple sum approach, two agencies could both receive a high score if they are doing many enforcement activities, but the specific activities they are doing could be very different. Using latent class analyses, we were able to identify qualitatively distinct patterns of enforcement activity. If specific patterns of enforcement are found to be more predictive of lower alcohol rates of alcohol use or related problems than other patterns, then advocates can promote use of that combination of enforcement strategies in their communities and states rather than simply advocating generally for more enforcement. This efficiency is particularly important when resources are limited.

However, one of the most compelling findings when examining results across the five enforcement domains is the sheer number of agencies that are in classes characterized by little or no enforcement. Slightly more than $60 \%$ of agencies are in the low enforcement class for the underage sale domain, meaning no compliance checks were conducted among these agencies. Similarly, nearly $60 \%$ of agencies were in the Uniformly Low class for the underage provision domain, and almost $80 \%$ of agencies were in the Uniformly Low class for the overservice domain. The large proportion of agencies in these low enforcement classes is particularly concerning as these three domains represent enforcement aimed at reducing the availability of alcohol, both for youth (social and commercial sources) and for intoxicated patrons at alcohol establishments. Reducing availability of alcohol has been shown to be a key strategy in prevention of alcohol-related problems (Babor et al., 2010; Hingson et al., 2005; Holder et al., 2000).
The complementary finding is that few agencies are in high enforcement classes. In four domains we identified a Uniformly High enforcement class, but the proportion of agencies in these classes were modest. Approximately 6\% of agencies were in the Uniformly High class for overservice, $20 \%$ for impaired driving, $22 \%$ for underage possession/consumption, and $26 \%$ for underage sales. For the underage provision class, there was no Uniformly High enforcement class. In this domain, not only are few agencies conducting underage provision enforcement activities, but educational efforts, which are not a direct enforcement activity, account for a fair proportion of the underage provision enforcement. One possible reason for the low prevalence of agencies doing high levels of these activities may be because the actions are covered by statelevel agencies. This may be particularly true for the impaired driving domain, where the state patrol may handle much of the enforcement. Further analyses combining state- and local-level enforcement data are needed to explore this hypothesis.

The underage sales enforcement domain is noteworthy as it has the most polarized pattern of activities compared to the other domains. More than $60 \%$ of agencies were in the low enforcement class (conducting no compliance checks) and $26 \%$ of agencies were in the high enforcement class (conducting fairly comprehensive compliance checks). Only $14 \%$ of agencies were in the middle classes, where compliance checks are conducted but either not uniformly across all premises in the jurisdiction or with limited frequency of checks or follow-ups. The positive implication is that once agencies commit to conducting compliance checks, the majority seem to do them fairly well. This is likely influenced by the availability of recommendations for agencies on how to implement alcohol compliance checks (www.epi.umn.edu/alcohol/ manual/index.shtm; www.pire.org/documents/ReduceAlsal. pdf; http://www.ptb.state.il.us/pdf/alcohol.pdf). The negative implication is that there are still a large number of agencies that are not doing any compliance checks. 
Analyses of the differences in community and agency characteristics between classes provide insight into what factors might influence the likelihood of an agency using different types of enforcement activities. In terms of community characteristics, few differences emerge. Although one might speculate that agencies in areas with smaller populations or more economic disadvantage may engage in fewer activities because of limited resources, we found little if any evidence that agencies in these communities differed in their activities from agencies in other types of jurisdictions. Two agency characteristics were consistently associated with class across domains. Agencies that had an officer assigned specifically to alcohol enforcement and agencies that perceived underage drinking, impaired driving, or overservice as more common were more likely to be in a class characterized by higher levels of enforcement. Unfortunately, it is difficult to fully interpret this association using only cross-sectional data; having an alcohol officer could lead to increased alcoholrelated enforcement or increased alcohol-related enforcement could lead to the addition of an alcoholspecific officer. Similarly, perception of alcohol problems could increase enforcement or increased enforcement could affect perceptions of alcohol problems. Longitudinal analyses are needed to better understand the direction of effects.

The current study has a number of limitations. First, the study relies on self-reported data from enforcement agencies, which may introduce socially desirable response bias. However, given that many of our participants reported that their agencies were not conducting many of the enforcement activities, this potential bias may be modest. Second, we surveyed only one officer from each enforcement agency and this person may not have been knowledgeable about all aspects of alcohol enforcement activities conducted by his or her agency. To minimize this potential problem, we attempted to survey the individual most knowledgeable about alcohol enforcement within each agency. Third, we attempted to obtain a representative sample of local law enforcement agencies from each state, but agencies that did not respond to our survey may have been less likely than agencies that did to engage in enforcement activities. Missing data examination showed few differences between responders and nonresponders. Finally, certain analytic simplifications were utilized. The data are hierarchical with agencies nested within states, but this was not accounted for in latent class models. Also, each agency was assigned to its most likely class and that assignment was used for the regression models. This does not account for differences in the probability of class membership; however, examination of the posterior probabilities suggests little concern.

Despite these limitations, this study is the first to examine patterns of alcohol enforcement activities across a national sample of local law enforcement agencies. The current analyses represent the first steps of identifying certain patterns of enforcement activities and community and agency characteristics associated with the use of these patterns. An important next step is to determine if these patterns are associated with state alcohol laws and rates of alcohol use and alcohol-related injury and mortality.

Results from this study are important to researchers, law enforcement, and advocates. For researchers, this study shows that latent class analyses can be used to identify patterns of enforcement strategies. Identification of enforcement patterns is important for future studies evaluating effects of combinations of enforcement strategies as well as for studies assessing effects of alcohol policies where controlling for complex enforcement activities would strengthen the study. For law enforcement and advocates, the results show a clear need for more alcohol-related enforcement strategies across many communities. The fact is that there are local law enforcement agencies across the U.S. of all sizes that are conducting alcohol-related enforcement, perhaps indicating that limited resources may not be the only reason for low levels of alcohol-related enforcement. Many of these agencies do not simply conduct one enforcement strategy to address a specific domain of alcohol-related problems, but rather use multiple strategies. This suggests that when there is a will (and potentially outside pressure), there is a way to find the necessary resources to address a range of alcohol-related problems.

\section{References}

Babor, T. F., Caetano, R., Casswell, S., Edwards, G., Giesbrecht, N., Grahm, K., . . . Rossow, I. (2010). Alcohol: No ordinary commodity: Research and public policy (2nd ed.). Oxford, England: Oxford University Press.

Collins, L. M., \& Lanza, S. T. (2010). Latent class and latent transition analysis: With applications in the social, behavioral, and health sciences. New York, NY, United States: John Wiley \& Sons, Inc.

Elder, R. W., Shults, R. A., Sleet, D. A., Nichols, J. L., Zaza, S., \& Thompson, R. (2002). Effectiveness of sobriety checkpoints for reducing alcohol-involved crashes. Traffic Injury Prevention, 3, 266-274.

Ferguson, S. A. (2012). Alcohol-impaired driving in the United States: Contributors to the problem and effective countermeasures. Traffic Injury Prevention, 13(5), 427-441.

Gibbs, J. P. (1975). Crime, punishment, and deterrence. New York, NY, United States: Elsevier.

Grube, J. W. (1997). Preventing sales of alcohol to minors: Results from a community trial. Addiction, 92(Suppl. 2), S251-S260.

Hingson, R. W., Zakocs, R. C., Heeren, T., Winter, M. R., Rosenbloom, D., \& DeJong, W. (2005). Effects on alcohol related fatal crashes of a community based initiative to increase substance abuse treatment and reduce alcohol availability. Injury Prevention, 11(2), 84-90.

Holder, H. D., Gruenewald, P. J., Ponicki, W. R., Treno, A. J., Grube, J. W., Saltz, R. F., . . Roeper, P. (2000). Effect of community-based interventions on high-risk drinking and alcohol-related injuries. Journal of the American Medical Association, 284(18), 2341-2347. 
Kerr, W. C. (2010). Categorizing U.S. state drinking practices and consumption trends. International Journal of Environmental Research and Public Health, 7, 269-283.

Muthén, L. K., \& Muthén, B. O. (1998-2012). Mplus User's Guide (7th ed.). Los Angeles, CA: Muthén \& Muthén.

National Research Council \& Institute of Medicine. (2004). Reducing underage drinking: A collective responsibility. Washington, DC, United States: The National Academies Press.

Ross, H. L. (1984). Social control through deterrence: Drinking-and-driving laws. Annual Review of Sociology, 10, 21-35.

SAS Institute, Inc. (Ed.). (2011). SAS/STAT 9.3 User's guide. Cary, NC, United States: SAS Institute, Inc.

Tittle, C. R. (1980). Sanctions and social deviance: The question of deterrence. New York, NY, United States: Praeger Publications.

Voas, R. B., Tippetts, A. S., \& Fell, J. C. (2003). Assessing the effectiveness of minimum legal drinking age and zero tolerance laws in the United States. Accident Analysis and Prevention, 35(4), 579-587.

Wagenaar, A. C., \& Toomey, T. L. (2002). Effects of minimum drinking age laws: Review and analyses of the literature from 1960 to 2000. Journal of Studies on Alcohol, Suppl. 14, 206-225.

Wagenaar, A. C., Toomey, T. L., \& Erickson, D. J. (2005). Complying with the minimum drinking age: Effects of enforcement and training interventions. Alcoholism, Clinical and Experimental Research, 29(2), 255-262. 